\title{
Analysis of Psychological Factors and Guidance Countermeasures of College Students' Using Behaviors of Social Network
}

\author{
Feifei Sun* \\ Zaozhuang University, Shandong, China \\ Sff_lovesea@126.com
}

Keywords: college students; social network; psychological factors; motivation; personality

\begin{abstract}
With the development of Internet technology and popularization of mobile terminals, the Internet across time and space has changed people's lifestyle gradually. Social network is the mobile terminal network application favored by college students. It brings not only the convenience but also some disadvantages for life and interpersonal communication of college students. Based on the psychological features of college students, this paper explores the psychological factors of college students' using behaviors of social network in order to guide college students to better use social network to broaden their knowledge and enhance friendship. This paper elaborates on the psychological factors of college students' using behaviors of social network from motivation and personality and proposes two suggestions of guiding college students to correctly use social network.
\end{abstract}

\section{Introduction}

With the development of Internet technology and popularization of mobile terminals, the utilization rate of social network is growing at a rapid speed. Social network has become one of the important tools for people in modern society to maintain, develop and expand social relations. College students are strongly curious about novel things with stronger ability to obtain new skills. According to research, around 1/3 of people who use social networks are college students [1]. College students have strong social and emotional needs, and the emergence of social network meets their needs and provides a good way for healthy mental and physical development of college students. However, there is a reason for the existence and development of any new thing. Besides, it will have an impact on different aspects. Social network can bring college students the satisfaction of social interaction as well as affect the life and social communication of college students.

\section{Analysis of Psychological Factors of College Students' Social Network Usage}

2.1 Analysis of motivation of using behaviors of social network. Motivation of social interaction. According to Maslow's hierarchy of needs theory, social interaction is one of the most basic needs of people. In the process of social interaction, obtaining the satisfaction of love and belonging is a necessary condition for physical and mental health. College students are in the age when they become alienated from the relationship with the primary family and focus more on the relationship with the peer relationship and the opposite sex. They are more willing to communicate and talk about life with their peers or the opposite sex. With the continuous development of Internet technology and the rapid popularization of mobile terminals, social network brings a brand new way of social interaction that changes our life.

In the context of lack of interpersonal communication, the use of social networks can obtain emotional and social support, which is very obvious among college students, especially among the freshmen. Freshmen enter the strange environment full of strangers from the environment full of familiar family members and friends. They are lack of interpersonal relationships with only a few roommates or classmates, which will make them panic or anxious. At this time, the use of social network can provide them with the interpersonal interaction model across time and space and fill in the blanks of freshmen's interpersonal interaction. It can greatly ease their negative emotions. College students pay more attention to communication with the opposite sex. It is obvious that 
college students have stronger needs for interaction with the opposite sex. Social network increases the opportunities for college students to meet the opposite sex, especially among the engineering and art majors with serious gender imbalance.

Motivation of information acquisition. The trans-time-space, convenience and rapid speed of social network provides information communication among the group with the best path. In modern universities, teaching and student management greatly depends upon social network. Various QQ groups and WeChat groups connect the members in various groups. These groups allow people to have access to school information or classroom information whenever and wherever they like. College students are used to sharing resources or collecting information by WeChat, Weibo and other social networks. Research shows that a lot of college students share class notes, homework and other learning information by group sharing and obtain various knowledge and social news by scanning articles and news. The immediacy of social network provides resource sharing and information collection with the fastest way.

Motivation of entertainment. Universities provide college students with more free and independent space. For those college students who can arrange their life and study in a planned and reasonable way, the entertainment function of social network brings them a way to relax themselves in hard work. However, such a free and independent status will bring more disadvantages for college students with poor self-management and obscure self-development objectives. Some college students do not know how to spend their time, and some of them realize the importance of independent study but fail to make a decision or keep their promise as they have no clear goal. When the college students do not know how to spend their time or avoid the psychological pressure brought by "self-decadence", they play games, chat with strangers online or scan social news aimlessly in order to kill time or obtain temporary spiritual relaxation through social networks [2].

2.2 The social network use behavior of the personality factor analysis. Factors of introversion and extraversion. Social network has changed the way that people socialize and provided college students of social anxiety with a more receptive way of social interaction [3]. Online social interaction can be controlled and delayed. It can be controlled means that there is no language clue in social activities through social network, and the text can be modified or deleted at any time. Besides, people can think about or modify what they want to express and control their expression in online social interaction. It can be delayed means that compared with timely offline social interaction, social activities through social network allow people to have enough time to think about how to reply to maintain a good self-image. It is a reliable and receptive way of social interaction for college students who are not good at social communication with a lack of experience in social experience or who worry about being rejected during social interaction. Thus, college students are more willing to begin interpersonal interaction through online social network compared with offline social interaction. Online social interaction is more about the continuation and complement for offline social interaction for college students with active offline social interaction. Research shows that the way of online social interaction is similar to that of offline social interaction. That is to say, college students with active offline social interaction are active during online social interaction. However, college students with inactive offline social interaction are less active in online social interaction than those with active social interaction. But online interaction is more active than offline interaction with more real self-expression.

Factors of self-concept. Interpersonal interaction by online social network is more of the continuation of offline interpersonal relationships. College students present and express themselves by publishing status and articles. When their friends reply feedback information, they may feel others recognize and pay attention themselves. And further feel emotional support and belonging of community. Such the emotional satisfaction and sense of community can bring active self-concept with pleasant emotional experience. Self-concept can affect the subjective sense of happiness of individuals. When self-concept of college students is attacked by reality, self-expression by social network with feedback can make up for the negative impact of reality on self-concept, which is helpful for maintaining active self-concept to obtain more sense of happiness and favorable for healthy mental and physical development. 


\section{Guidance Countermeasures of College Students' Using Behaviors of Social Network}

\subsection{Correctly understand the deep psychological factors behind using behaviors of social} network. There are deep psychological factors in the occurrence of any behavior. Only by correctly understanding these deep psychological factors can we better understand and explain the behaviors. Good using behaviors of social network with a correct understanding of psychological factors of the occurrence can help college students present more good using behaviors of social network. Bad using behaviors of social network with a correct understanding of the psychological factors of bad using behaviors of social network will help them adjust behavioral cognition and reduce the bad using behaviors of social network. The typical bad using behavior of social network among college students is entertainment by frequent use of social network. According to investigation and interviews, college students avoid the negative emotions or pressure of real life by relying on or getting addicted to social network for entertainment. The ideal life status of these college students is very active. However, their real life is negative. They are to laze to get up, they have to stop in face of difficult problems and continue to paralyze themselves in social networks. It is greatly related to the lack of guidance in self-restriction, clear objectives and time management of college students. The lack of school guidance, teachers' guidance and students' self-reflection make some college students avoid reality in the virtual world of social network.

From the perspective of educators, schools can make college students realize their individual and social responsibilities as modern young people through lectures, reports and other forms so that they can enhance their sense of responsibility, feel the personal charm and pursuit of professionals in some fields and improve their desire for knowledge. Teachers can discover the strengths and weaknesses of college students as soon as possible through communications with them in order to correct and actively guide their shortcomings, promote their physical and mental health and enhance their self-cognition to help them overcome their weaknesses for continuous self-development and self-improvement.

College students should recognize themselves, overcome their weaknesses and become the master of life through various ways. College students should develop clear objectives in order to overcome difficulties, which is most important factor for college students to master their life. Their actions will stop without clear objectives, which is the major reason for some college students make the decision.

3.2 Improve the quality of life by rational use of interpersonal and knowledge resources of social networks. The rational use of social networks can enhance friendship. Developing active and healthy interpersonal relationships is one of the important indicators to enhance college students' happiness of college life. Social network maintains and broadens the social interaction circle of college students, continue offline friendship online, make strangers enter the circle of friends and increase social capitals [6]. However, online social networking cannot completely replace the interpersonal interaction in real life. There is no real emotion conveyed by living people through the Internet. Or the emotions interpreted by the Internet are greatly self-imagined without any warmth and with a lack of certain authenticity. Therefore, college students should realize that communication in real life is still the major way for social interaction. They should make reasonable allocation and control of their time in online social interaction.

The rational use of social networks can broaden knowledge. The Internet makes people enter the age of knowledge explosion. It is full of endless and vast knowledge. The rich information and convenient way to obtain knowledge makes human reception and dissemination of knowledge enters the fast-food age. Investigations show that modern college students prefer to obtain knowledge and news by social networks such as Weibo and WeChat rather than study cultural knowledge by thinking. Culture without the thinking of human beings is like a tree without root that cannot stand the wind and rain. How is Chinese deeply rooted culture carried forward in the age of fast-food culture? Thus, news and knowledge with stronger demand for immediacy can rely on convenient and rapid social networks so that college students can know about the society and reality at first time. The reading and thinking of deeply rooted culture still requires college students to get themselves immersed in thinking. Thus, they should reasonably allocate and control their time in 
social networks to obtain knowledge.

\section{Conclusion}

College students walk in the forefront of the times. As the extensive disseminator of new technology and new things, they shoulder social and national responsibilities. Therefore, it is the most important work of educators to guide college students comprehensively. The Internet age brings opportunities and challenges for the society and college students. It is an important part of modern higher education to guide college students to use network resources in a reasonable way, which educators should conduct in-depth exploration.

\section{References:}

[1] T. Li, Research of College Students' Use and Satisfaction of SNS Social Network Sites, vol.20, pp. 20-22, 2016.

[2] Y.ZH. Jiang, X.L.Bai, Teenagers' Problematic Use of Social Network, Advances in Psychological Science, vol.24, pp. 1435-1437, 2016.

[3] Y.F. Gong, Introversion and Extroversion of College Students-Research of the Relationship between Sense of Belonging and Use of Online Social Network, vol.15, pp. 6-8, 2016. 